\title{
Acidentes domésticos na infância: Identificando potencialidades para um cuidado integral
}

Domestic Accidents in childhood: Identifying potentialities for comprehensive care

Accidentes domésticos em la infancia: Identificación de potencialidades de atención integral

Recebido: 08/07/2021 | Revisado: 17/07/2021 | Aceito: 23/07/2021 | Publicado: 31/07/2021

Wallace Henrique Pinho da Paixão

ORCID: https://orcid.org/0000-0002-2533-4707 Instituto Nacional do Câncer, Brasil

E-mail: whpp2601@ @otmail.com

Kessya Cristina Valentim Barbosa

ORCID: https://orcid.org/0000-0002-8490-2575 Hospital Federal do Andaraí, Brasil

E-mail: kessyacvb@gmail.com

Jamilly Cristina Elias Pinheiro

ORCID: https://orcid.org/0000-0002-8890-5992

Hospital Federal dos Servidores do Estado, Brasil

E-mail: pinheiro_jamilly@yahoo.com.br

Kelly Cristina Freitas da Silva

ORCID: https://orcid.org/0000-0002-0882-8537

Instituto Nacional de Cardiologia, Brasil

E-mail: kecrisfreitas@gmail.com

Maria Regina Bernardo da Silva

ORCID: https://orcid.org/0000-0002-3620-3091

Secretaria Municipal de Saúde do Rio de Janeiro, Brasil

E-mail: m.regina2000@uol.com.br

Wanderson Alves Ribeiro

ORCID: https://orcid.org/0000-0001-8655-3789

Universidade Iguaçu, Brasil

Universidade Federal Fluminense, Brasi

E-mail: nursing_war@hotmail.com

Nathalya de Moura Rezende

ORCID: https://orcid.org/0000-0001-7972-0956

Instituto Nacional do Câncer, Brasil

E-mail: nathalyamrezende@gmail.com

Keila do Carmo Neves

ORCID: https://orcid.org/0000-0001-6164-1336

Universidade Iguaç, Brasil

Universidade Federal do Rio de Janeiro, Brasil

E-mail: keila_arcanjo@hotmail.com

Bruna Porath Azevedo Fassarella

ORCID: https://orcid.org/0000-0002-1400-4147

Universidade Iguaçu, Brasil

Universidade de Vassouras, Brasil

E-mail: brunaporath@gmail.com

Carolina Cristina Scrivano dos Santos

ORCID: https://orcid.org/0000-0003-2456-7178

Instituto Nacional do Câncer, Brasil

E-mail: carolcristina98@hotmail.com

\section{Resumo}

Os acidentes domésticos na infância (ADI) embora subestimados caracterizam-se como um relevante problema de saúde no mundo, devido à sua potencial gravidade. Segundo Ministério da Saúde em 2010 ocorreram 11,6 mil internações resultantes de acidentes no domicílio. Os profissionais de enfermagem dispõem de aptidão para executar ações educativas com os pais e/ou responsáveis e as crianças com o objetivo de evitar acidentes. Objetivos: identificar as principais características que cercam os ADI e qual é o papel do enfermeiro diante de tal problemática. Metodologia: Trata-se de uma revisão integrativa. Os critérios de inclusão foram: artigos com recorte temporal entre 2015 e 2020; nos idiomas português, inglês e espanhol. Foram utilizadas bases de dados: BVS, SciELO e MEDLINE através do PubMed. Foram selecionados 13 artigos para discussão. Resultados: Foram identificados 10 estudos nacionais e 3 internacionais. Quanto ao tipo, 10 são originais, dois são de revisão e um relato de experiência. Os principais ADI evidenciados foram: quedas, queimadura, asfixia, corte e intoxicação, mordida de cachorro e 
afogamento. Discussão: Para melhor compreensão acerca da temática, a discussão foi dividida em quatro categorias, a saber: Epidemiologia dos ADI, fatores de risco, perfil familiar e das crianças e a assistência de enfermagem na prevenção dos ADI. Conclusão: Diversos são os fatores de risco presentes no ambiente doméstico, todavia, muitos desses fatores podem ser reduzidos e até eliminados, a partir da conscientização dos pais e cuidadores. O enfermeiro possui essencial atuação na educação de pais e familiares na prevenção dos ADI.

Palavras-chave: Acidentes domésticos; Crianças; Fatores de risco; Enfermagem.

\begin{abstract}
Domestic accidents in childhood (DAC), although underestimated, are characterized as a relevant health problem in the world, due to their potential severity. According to the Ministry of Health in 2010, there were 11,600 hospitalizations resulting from accidents at home. Nursing professionals have the ability to carry out educational actions with parents and / or guardians and children in order to avoid accidents. Objectives: to identify the main characteristics that surround DAC and what is the role of the nurse in the face of such problem. Methodology: This is an integrative review. The inclusion criteria were: articles with a time frame between 2015 to 2020; in Portuguese, English and Spanish. Databases were used: VHL, SciELO and MEDLINE through PubMed. 13 articles were selected for discussion. Results: 10 national and 3 international studies were identified. As for the type, 10 are original, 2 are for review and 1 is an experience report. The main ADI evidenced were: falls, burn, asphyxiation, cut and intoxication, dog bite and drowning. Discussion: For a better understanding of the theme, the discussion was divided into four categories, namely: Epidemiology of DAC, risk factors, family and children's profile and nursing care in the prevention of DAC. Conclusion: There are several risk factors present in the domestic environment, however, many of these factors can be reduced and even eliminated, based on the awareness of parents and caregivers. The nurse has an essential role in the education of parents and family in the prevention of DAC.
\end{abstract}

Keywords: Domestic accidents; Kids; Epidemiology; Risk factors; Nursing.

\title{
Resumen
}

Los accidentes domésticos en la infancia (ADI), aunque subestimados, se caracterizan como un problema de salud relevante en el mundo, por su potencial gravedad. Según el Ministerio de salud en 2010 hubo 11.600 hospitalizaciones por accidentes en el hogar. Los profesionales de enfermería tienen la capacidad de realizar acciones educativas con padres y/o tutores y niños con el fin de evitar accidentes. Objetivos: Identificar las principales características que rodean a la ADI y cuál es el rol del enfermero ante tal problema. Metodologia: Esta es una revisión integradora. Los criterios de inclusión fueron: artículos con un marco temporal entre 2015 y 2020; en português, inglés y espanhõl. Se utilizaron bases de datos: BVS, SciElo y MedLINE através de PubMed. Se seleccionaron 13 artículos para discusión. Resultados: Se identificaron 10 estudios nacionales u 3 internacionales. En cuantoal tipo, 10 son originales, 2 son para revisión y 1 es un informe de experiencia. Las principales ADI evidenciadas fueron: caídas, quemaduras, asfixia, cortes e intoxicación, mordedura de perro y ahogamiento. Discussión: Para uns mejor comprensión del tema, la discusión se dividió en cuatro categorías, a saber: Epidemiologia de la ADI, factores de riesgo, perfil familiar y del niño y cuidados de enfermería en la prevención de la ADI. Conclusión: Existen varios factores de riesgo en el entorno doméstico, sin enbargo, muchos de estos factores pueden reducirse e incluso eliminarse, en base a la conciencia de los padres y cuidadores. La enfermera tiene un papel fundamental en la educación de los padres y la familia en la prevención de la ADI.

Palabras clave: Accidentes domésticos; Niños; Epidemiología; Factores de riesgo; Enfermería.

\section{Introdução}

A Organização Mundial de Saúde (OMS) define como acidente "um acontecimento independente da vontade humana.”, contudo, o padrão usual que o compreende como inevitável e imprevisível vem sendo transformado e uma nova concepção conceitua o acidente como um episódio previsível, sendo capaz de gerar lesões ou até o óbito. (Batalha et al., 2016) Os acidentes domésticos na infância (ADI), embora subestimados, caracterizam-se como um relevante problema de saúde no mundo, devido à sua potencial gravidade (Silva et al., 2017).

Nesse sentido, ocorrência de ADI possui relação direta com o comportamento familiar e meio social, estando intimamente relacionada a hábitos de vida, condições educacionais, econômicas, sociais e culturais, associada à idade específica das crianças, somada à curiosidade e desejo de aprender (Moita et al., 2018).

Se tratando de segurança contra desastres e incidentes, quanto mais nova e imatura a criança for, menos compreensão de risco ela terá e mais vulnerável e dependente de outras pessoas ela será. Até os quatro anos, apresenta-se curiosa, confunde o real e o imaginário e reproduz ações dos adultos. Após os quatro anos são instigados pelos desafios. Ressalta-se a facilidade 
para queda devido à desproporcionalidade da cabeça em relação ao corpo, sendo o peito o centro da gravidade, ao invés do umbigo. Somente após os sete anos, desenvolve-se o discernimento para identificar riscos e esses fatores devem ser de conhecimento dos pais para promover maior segurança aos filhos (Gomes et al., 2013).

Segundo Ministério da Saúde em 2010 ocorreram 11,6 mil internações resultantes de acidentes no domicílio, onde as principais vítimas eram maiores de um ano e o percentual de meninos foi maior do que o de meninas (Brasil, 2012).

Ao avaliar os atendimentos em unidades de urgência e emergência em 24 capitais brasileiras e no Distrito Federal, constatou que das $67,9 \%$ das 7.224 crianças de 0 a 9 anos internadas por acidentes, foram no domicílio. Os AD são situações complexas, evitáveis em sua maioria. ${ }^{7}$ Além de causarem prejuízos sociais, econômicos e emocionais, também resultam em sequelas e eventos fatais que, futuramente, reverberam na família e na sociedade, contristando as crianças e adolescentes (Lima et al., 2018).

Algumas condições foram caracterizadas o risco de acidentes infantis, como os referentes a alicerce familiar que incluem os pais solteiros e questões socioeconômicos onde a família com menores condições financeiras supervisam os filhos de modo inadequado, deixando-os sob incumbência de vizinhos e parentes (Ribeiro et al., 2019).

As melhores maneiras de atuar no desenvolvimento da conscientização se dão a partir de condutas preventivas, que norteiam cuidados físicos, materiais e emocionais, (Correa et al., 2006) intervindo desde a criação de programas de educação com início no ensino pré-escolar e perto da residência, até a execução de normas e medidas de proteção, sendo consideradas ações eficientes (Batalha et al., 2016).

Para defrontar um problema de ampla magnitude, meios de imersão nas regiões com o objetivo de analisar e entender as necessidades de saúde da população e comunidades são uma boa estratégia para a implantação de intervenções de promoção à saúde (Lima et al., 2013). Uma vez que a comunidade se mostra participativa, abordar a família pode ser eficiente no sentido de diminuir as ocorrências de acidentes (Acker \& Cartana, 2009).

Os profissionais de enfermagem dispõem de aptidão para executar ações educativas com os pais e/ou responsáveis e as crianças com o objetivo de evitar acidentes. As instruções transitam pelo caráter preventivo e de promoção do bem-estar na infância e o compromisso com de adesão a hábitos adequados. A equipe de enfermagem que atua em escolas, creches, ambulatórios e unidades de saúde possuem maior privilégio em termos de oportunidade para conscientização envolvendo a prevenção de acidentes. Ademais, o trabalhador também deve ter entendimento sobre os acidentes na infância em todas as fases, para que em conjunto com os pais, possam articular maior domínio sobre as tais questões (Moita et al., 2018).

Diante disso, tivemos como objetivos: identificar as principais características que cercam os ADI e qual é o papel do enfermeiro diante de tal problemática.

\section{Metodologia}

Trata-se de uma revisão integrativa da literatura de natureza qualitativa. O estudo qualitativo verifica o acontecimento no âmbito natural, sendo também denominada como "naturalística" (Teis\&Teis, 2006) e buscou apresentar a revisão e análise crítica acerca da temática dos ADI. Para sua construção determinou-se a divisão em 5 etapas, sendo elas: Identificação do problema; busca na literatura/coleta de dados; verificação dos dados; análise dos conteúdos encontrados nos artigos incluídos na revisão; exposição e interpretação dos resultados.

Na primeira etapa, foi determinada a identificação do problema. Para isso, listaram-se as principais percepções sobre a associação entre os acidentes domésticos e variáveis definidoras para o acontecimento do evento. Devido a isso, o trabalho foi conduzido com a seguinte questão norteadora: Quais são os fatores que propiciam a ocorrência de acidentes domésticos na infância? E para a criação da pergunta de pesquisa, utilizou-se a estratégia PICo (Quadro 1), onde o P corresponde aos Participantes, I ao fenômeno de Interesse e Co ao Contexto do estudo (Ribeiro et al., 2019). 
Quadro 1 - Estratificação da pergunta de pesquisa seguindo a estratégia PICo (Ribeiro et al., 2019; Karino e Felli 2012).

\begin{tabular}{|c|c|c|c|c|}
\hline DESCRIÇÃO & PICo & COMPONENTES & DESCRITORES & TIPO \\
\hline PARTICIPANTES & $\mathrm{P}$ & CRIANÇAS & CRIANÇAS & DeCS \\
\hline FENÔMENO & $\mathrm{I}$ & FATORES & ENFERMAGEM & DeCS \\
DE INTERESSE & & ENVOLVIDOS & FATORES DE RISCOS & \\
\hline $\begin{array}{c}\text { CONTEXTO } \\
\text { DO ESTUDO }\end{array}$ & Co & ACIDENTES & ACIDENTES DOMÉSTICOS & DeCS \\
& & DOMÉSTICOS & & \\
\hline
\end{tabular}

Fonte: Autores.

A segunda etapa iniciou-se com a determinação dos descritores, a partir dos Descritores em Ciências da Saúde (DeCS). Foram selecionados os seguintes descritores: Acidentes domésticos; Crianças; Epidemiologia; Fatores de risco; Enfermagem. Todos utilizados em conjunto a partir do boleanoAND.

Após definidos os descritores, iniciou-se a busca na literatura. Foram utilizados como critérios de inclusão: artigos com recorte temporal entre 2015 a 2020; nos idiomas português, inglês e espanhol; com resumo disponível da base de dados selecionada e texto completo disponível. Foram excluídos estudos que abordassem outra faixa etária senão a infantil, além de outros tipos de acidentes (transito).

A busca na literatura ocorreu entre os meses de Maio e Agosto de 2020. Foram utilizadas bases de dados Biblioteca Virtual de Saúde (BVS), ScientificElectronic Library Online (SciELO) e Literatura Internacional em Ciências da Saúde (MEDLINE) através do PubMed.

A seleção dos artigos teve seu início a partir da inserção dos descritores nas bases de dados supracitados. Foram encontrados ao todo, 7.555 artigos. Por conseguinte, foi realizada a filtragem através da própria base de dados, adicionando os critérios de inclusão descritos anteriormente, restando 794 artigos. Cabe destacar que desses, 48 apresentavam-se duplicados nas bases de dados, onde, depois de excluídos restaram 746 artigos. Por conseguinte, se deu início a leitura dos títulos e resumos. Após uma criteriosa leitura dos títulos e resumos, foram excluídos 688 artigos, pois não correspondiam aos critérios de elegibilidade. Restou-se então, 58 artigos para leitura completa. Após criteriosa leitura dos artigos na íntegra, 45 não corresponderam aos critérios de elegibilidade, sendo selecionados 13 artigos conforme evidenciado na Figura 1, seguindo recomendações de fluxograma PRISMA. 
Figura 1: Fluxograma de quantitativo inicial de artigos encontrados nas bases de dados científicas até a sua seleção final, de acordo com recomendação do Preferred Reporting Items for Reviews and Meta-Analyses (PRISMA).

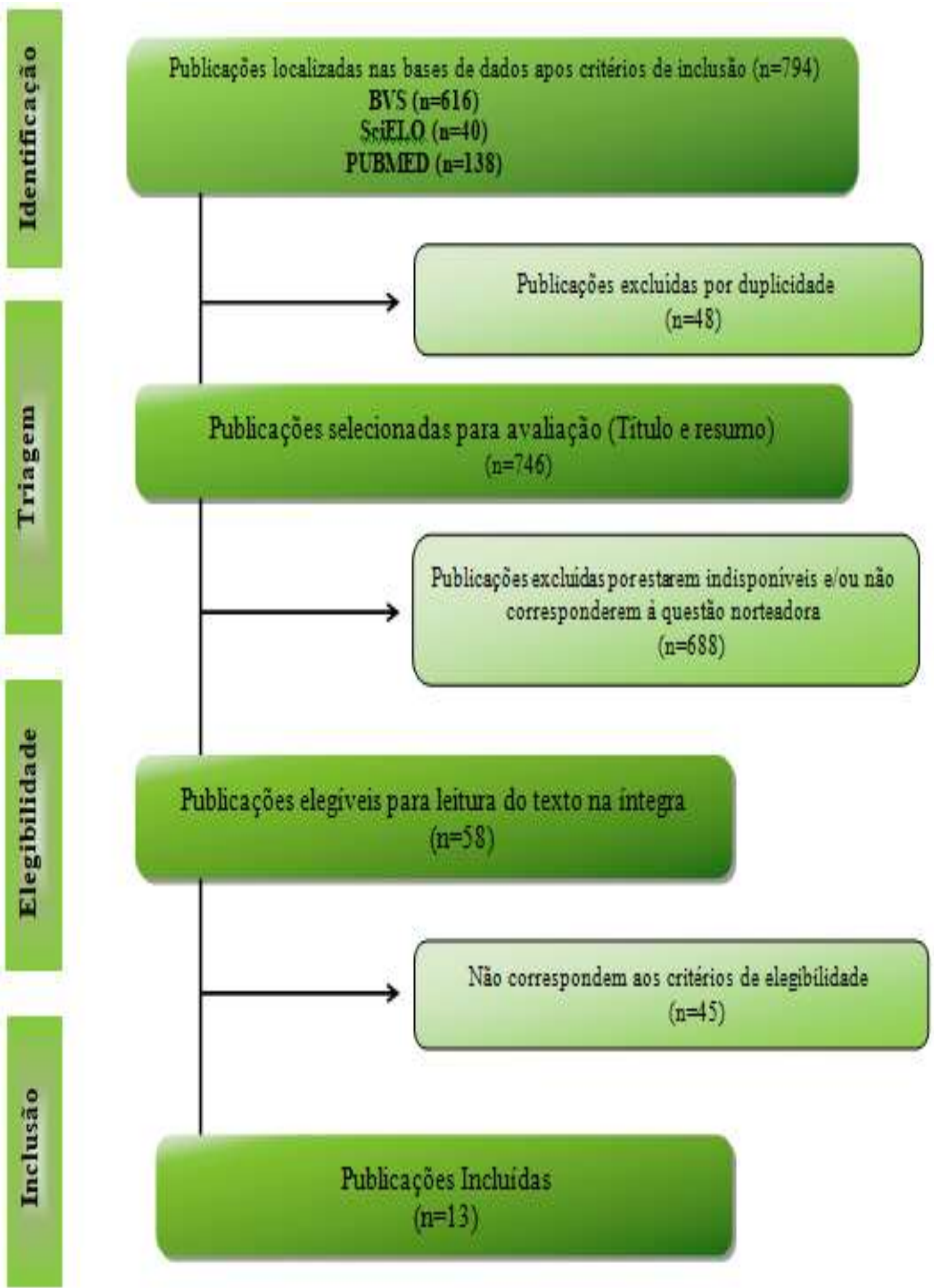

Fonte: Autores. 
Quadro 2: Quadro sinóptico com descrição das características de cada estudo.

\begin{tabular}{|c|c|c|c|c|}
\hline REVISTA & TÍTULO & $\begin{array}{c}\text { AUTOR E } \\
\text { ANO }\end{array}$ & OBJETIVOS & MÉTODOS \\
\hline $\begin{array}{l}\text { Revista } \\
\text { Gaúcha de } \\
\text { Enfermage } \\
\text { m }\end{array}$ & $\begin{array}{l}\text { Fatores de risco no ambiente } \\
\text { doméstico para } \\
\text { quedas em crianças menores } \\
\text { de cinco anos. }\end{array}$ & $\begin{array}{l}\text { Brito et al. } \\
2017\end{array}$ & $\begin{array}{l}\text { Analisar os fatores de risco no ambiente } \\
\text { doméstico para a ocorrência de quedas } \\
\text { em crianças menores de cinco anos }\end{array}$ & $\begin{array}{l}\text { Estudo transversal realizado em maio e junho de } 2016 \text { com } 344 \text { cuidadores de crianças do } \\
\text { Nordeste do Brasil. Utilizou-se um formulário referente às questões socioeconômicas e um check- } \\
\text { list para observação do ambiente. Realizaram-se análises bivariadas, utilizando-se teste qui- } \\
\text { quadrado de Pearson. }\end{array}$ \\
\hline $\begin{array}{l}\text { Journal of } \\
\text { Research } \\
\text { Fundament } \\
\text { al Care } \\
\text { Online }\end{array}$ & $\begin{array}{l}\text { Accident victim of domestic } \\
\text { child under the look of } \\
\text { nursing theories. }\end{array}$ & $\begin{array}{l}\text { Brito \& Rocha } \\
2015\end{array}$ & $\begin{array}{l}\text { Mostrar, a partir da produção científica, } \\
\text { os acidentes domésticos com crianças e } \\
\text { correlacioná-los às Teorias de } \\
\text { Enfermagem. }\end{array}$ & $\begin{array}{l}\text { Trata-se de uma revisão integrativa, realizada em julho de 2013, nas bases de dados: LILACS, } \\
\text { SCIELO, BDENF e MEDLINE. Adotaram-se como critérios de inclusão: pesquisas realizadas que } \\
\text { trabalhassem com o tema; disponíveis na íntegra; em língua portuguesa, espanhola ou inglesa, } \\
\text { publicadas entre } 2000 \text { e 2013, sendo excluídos: trabalhos monográficos e resumos. }\end{array}$ \\
\hline $\begin{array}{l}\text { Revista de } \\
\text { Enfermage } \\
m \text { UFPE } \\
\text { online. }\end{array}$ & $\begin{array}{lcc}\text { Perfil de } & \text { Crianças } & \text { e } \\
\text { Adolescentes } & \text { Internados } & \text { no } \\
\text { Centro de } & \text { Terapia } & \text { de } \\
\text { Queimados. } & & \end{array}$ & $\begin{array}{l}\text { Correia et al., } \\
2019\end{array}$ & $\begin{array}{l}\text { Descrever o acidente e o perfil de } \\
\text { crianças e adolescentes vítimas de } \\
\text { queimaduras internados em um Centro de } \\
\text { Terapia de Queimados de um Hospital } \\
\text { Geral. }\end{array}$ & $\begin{array}{l}\text { de prontuário de internos na faixa etária de um dia a } 18 \text { anos, de } 2014 \text { a } 2 \\
\text { responsáveis pelos internos, e apresentaram se os resultados em forma de ta }\end{array}$ \\
\hline $\begin{array}{l}\text { Bull } \\
\text { Emerg } \\
\text { Trauma }\end{array}$ & $\begin{array}{l}\text { Domestic Injuries among } \\
\text { Children Under } 7 \text { Years of } \\
\text { Age in Iran; The Baseline } \\
\text { Results from the Iranian First } \\
\text { Registry. }\end{array}$ & $\begin{array}{l}\text { Esfanjani } \quad \text { et } \\
\text { al., } 2017\end{array}$ & $\begin{array}{l}\text { Investigar aspectos epidemiológicos de } \\
\text { lesões em crianças iranianas menores de } 7 \\
\text { anos de idade usando dados obtidos em } \\
\text { um registro nacional. }\end{array}$ & $\begin{array}{l}\text { Os dados de lesões foram derivados de um sistema nacional de supervisão de lesões durante o } \\
\text { período } 2000-2002 \text {. Esse registro envolveu todas as lesões relacionadas à casa de crianças com } \\
\text { menos de } 7 \text { anos de idade, tratadas em centros de saúde ou de emergência. A população do estudo } \\
\text { incluiu } 25 \% \text { da população iraniana. Os métodos estatísticos descritivos foram utilizados para } \\
\text { representar a distribuição das variáveis como idade, sexo, mecanismos de lesão, tipos de lesões, } \\
\text { etc. Os dados foram apresentados como média } \pm \text { DP e proporções, conforme apropriado. }\end{array}$ \\
\hline $\begin{array}{l}\text { Rev. } \\
\text { Enfermería } \\
\text { Universitar } \\
\text { ia }\end{array}$ & $\begin{array}{l}\text { Prevalencia de accidentes en } \\
\text { el hogar en niños y factores } \\
\text { de riesgo associados. }\end{array}$ & 2015 & $\begin{array}{l}\text { Identificar a prevalência de acidentes em } \\
\text { crianças, em casa, e fatores de risco } \\
\text { associados. }\end{array}$ & $\begin{array}{l}\text { Estudo transversal descritivo, no período de agosto de } 2009 \text { a julho de } 2010 \text {. Foi realizada uma } \\
\text { amostragem não probabilística por conveniência, sendo aplicado um questionário estruturado aos } \\
\text { pais ou cuidadores de crianças menores de } 5 \text { anos que, durante o período do estudo, compareceram } \\
\text { a uma unidade de atendimento médico de nível primário do Instituto Mexicano de Seguridade } \\
\text { Social no DF, México. }\end{array}$ \\
\hline $\begin{array}{l}\text { Enfermage } \\
\mathrm{m} \text { em Foco }\end{array}$ & $\begin{array}{l}\text { Identificação dos } \\
\text { conhecimentos de mães na } \\
\text { prevenção de acidentes } \\
\text { domésticos com crianças da } \\
\text { primeira infância. }\end{array}$ & $\begin{array}{l}\text { Lima et al., } \\
2018\end{array}$ & $\begin{array}{l}\text { Identificar os conhecimentos das mães } \\
\text { sobre a prevenção de acidentes } \\
\text { domésticos infantis com crianças da } \\
\text { primeira infância. }\end{array}$ & $\begin{array}{l}\text { Estudo descritivo-exploratório, com abordagem quantitativa. Foi realizado em um ambulatório de } \\
\text { puericultura localizado no Campus do Pici da Universidade Federal do Ceará, na cidade de } \\
\text { Fortaleza. A amostra foi constituída por } 32 \text { mães. Resultados: o estudo demonstrou que há } \\
\text { predominância de acidentes domésticos infantis em populações de baixa renda, escolaridade } \\
\text { média, idade jovem e com várias pessoas morando na mesma residência. }\end{array}$ \\
\hline $\begin{array}{l}\text { Revista de } \\
\text { Enfermage } \\
\mathrm{m} \text { e } \\
\text { Atenção à } \\
\text { Saúde }\end{array}$ & $\begin{array}{l}\text { A importância da prevenção } \\
\text { de acidentes na infância: } \\
\text { Relato de experiência. }\end{array}$ & $\begin{array}{lr}\text { Margotti } & \text { et } \\
\text { al., } & 2018\end{array}$ & $\begin{array}{l}\text { Relatar a experiência vivenciada por } \\
\text { acadêmicos de enfermagem na realização } \\
\text { de ações educativas do projeto de } \\
\text { extensão "Acidentes domésticos na } \\
\text { infância não é brincadeira" }\end{array}$ & $\begin{array}{l}\text { Trata-se de relato de experiência, vivenciado por acadêmicos de enfermagem, do sexto período, na } \\
\text { realização do projeto de extensão apoiado pela Universidade Federal do Pará, executando ações } \\
\text { educativas para orientar crianças, pais ou cuidadores, sobre prevenção de acidentes domésticos na } \\
\text { infância, utilizando-se do lúdico, em especial do teatro de fantoches. }\end{array}$ \\
\hline
\end{tabular}




\begin{tabular}{|c|c|c|c|c|}
\hline $\begin{array}{l}\text { Revista } \\
\text { Portuguesa } \\
\text { de } \\
\text { Medicina } \\
\text { Geral e } \\
\text { Família }\end{array}$ & $\begin{array}{l}\text { Conhecimentos e práticas } \\
\text { parentais sobre medidas } \\
\text { preventivas de acidentes } \\
\text { domésticos e de viação. }\end{array}$ & $\begin{array}{l}\text { Ribeiro et al., } \\
2019 \text { (A) }\end{array}$ & $\begin{array}{l}\text { Avaliar os conhecimentos e práticas dos } \\
\text { pais sobre medidas preventivas de } \\
\text { acidentes domésticos e de viação. }\end{array}$ & $\begin{array}{l}\text { Aplicação de um questionário preenchido pelos pais das crianças observadas na consulta de Saúde } \\
\text { Infantil e Juvenil,entre maio e julho de } 2016 \text {. O questionário incluiu a caracterização sociofamiliar, } \\
\text { a avaliação dos conhecimentos sobre o tema, as medidas de prevenção utilizadas e a história } \\
\text { pessoal de acidentes. A análise estatística dos dados foi realizada através do programa SPSS 21.0. }\end{array}$ \\
\hline $\begin{array}{l}\text { Revista } \\
\text { Brasileira } \\
\text { de } \\
\text { Enfermage } \\
m\end{array}$ & $\begin{array}{l}\text { Determinantes sociais da } \\
\text { saúde associados a acidentes } \\
\text { domésticos na infância: uma } \\
\text { revisão integrativa }\end{array}$ & $\begin{array}{l}\text { Ribeiro et al., } \\
2019 \text { (B) }\end{array}$ & $\begin{array}{l}\text { Analisar os fatores associados aos } \\
\text { acidentes domésticos na infância segundo } \\
\text { os níveis dos determinantes sociais da } \\
\text { saúde }\end{array}$ & $\begin{array}{l}\text { Revisão integrativa da literatura, com pesquisa em bases de dados CINAHL, LILACS e PubMed, } \\
\text { com os seguintes descritores principais: child; social determinants of health; accidentes, home. } \\
\text { Foram incluídos } 31 \text { estudos que relacionaram os determinantes sociais da saúde e acidentes } \\
\text { domésticos na infância, em inglês, português e espanhol. }\end{array}$ \\
\hline $\begin{array}{l}\text { Revista } \\
\text { Baiana de } \\
\text { Enfermage } \\
\text { m }\end{array}$ & $\begin{array}{lr}\text { Intoxicação } & \text { na primeira } \\
\text { infância: } & \text { socorros } \\
\text { domiciliares } & \text { realizados por } \\
\text { adultos. } & \end{array}$ & $\begin{array}{l}\text { Sales et al., } \\
2017\end{array}$ & $\begin{array}{l}\text { Identificar a presença e as ações de } \\
\text { adultos no local da ocorrência de } \\
\text { acidentes toxicológicos infantis e os } \\
\text { primeiros socorros realizados. }\end{array}$ & $\begin{array}{l}\text { Estudo transversal, com análise retrospectiva de fichas de ocorrência toxicológica de crianças de } \\
\text { zero a quatro anos, arquivadas em um centro de assistência toxicológica. }\end{array}$ \\
\hline $\begin{array}{l}\text { Revista } \\
\text { Brasileira } \\
\text { de } \\
\text { Queimadur } \\
\text { as. }\end{array}$ & $\begin{array}{l}\text { Perfil epidemiológico de } \\
\text { crianças atendidas em uma } \\
\text { Unidade de Tratamento de } \\
\text { Queimados no interior de } \\
\text { São Paulo. }\end{array}$ & $\begin{array}{l}\text { Sanches et al., } \\
2016\end{array}$ & $\begin{array}{l}\text { Verificar a etiologia e a idade das } \\
\text { crianças internadas na Unidade de } \\
\text { Tratamento de Queimados de Catanduva, } \\
\text { SP. }\end{array}$ & $\begin{array}{l}\text { Foram avaliados } 190 \text { prontuários de crianças de } 0 \text { a } 15 \text { anos, internadas no período entre janeiro de } \\
2012 \text { e dezembro de } 2015 \text {. }\end{array}$ \\
\hline $\begin{array}{l}\text { Journal of } \\
\text { Human } \\
\text { Growth an } \\
\text { d Develop } \\
\text { ment. }\end{array}$ & $\begin{array}{l}\text { Fatores determinantes para a } \\
\text { ocorrência de }\end{array}$ & $\begin{array}{l}\text { Silva et al., } \\
2017\end{array}$ & $\begin{array}{l}\text { Analisar os fatores determinantes para } \\
\text { ocorrência de acidentes domésticos na } \\
\text { primeira } \\
\text { infância. }\end{array}$ & $\begin{array}{l}\text { Trata-se de um estudo exploratório e descritivo, transversal, realizado no município de Teresina- } \\
\text { PI, no Hospital de Urgência, no período de janeiro a março de 2016. A população foi composta por } \\
21 \text { cuidadores de crianças menores de cinco anos internados entre janeiro a março de } 2016 \text {. Para } \\
\text { coleta de dados realizou-se uma entrevista estruturada sobre os aspectos relacionados aos fatores } \\
\text { determinantes para ocorrência de acidentes domésticos com crianças. Realizou-se uma análise } \\
\text { descritiva, a partir de frequências absolutas e relativas para as variáveis. }\end{array}$ \\
\hline $\begin{array}{l}\text { Revista } \\
\text { Acta } \\
\text { Paulista de } \\
\text { Enfermage } \\
\mathrm{m}\end{array}$ & $\begin{array}{l}\text { Intervenção educativa com } \\
\text { mães jovens: aquisição } \\
\text { de saberes sobre cuidados da } \\
\text { criança. }\end{array}$ & $\begin{array}{l}\text { Silva et al., } \\
2018\end{array}$ & $\begin{array}{l}\text { Identificar os saberes das mães jovens } \\
\text { sobre o cuidado cotidiano da criança a } \\
\text { partir de intervenção educativa, em busca } \\
\text { de } \\
\text { ao cuidado integral à saúde. }\end{array}$ & $\begin{array}{l}\text { Estudo descritivo e de intervenção, desenvolvido com } 20 \text { mães entre } 16 \text { e } 25 \text { anos de idade com } \\
\text { filhos menores de três anos, pertencentes à área de abrangência de uma unidade de saúde da } \\
\text { família. A intervenção educativa foi baseada em cinco dinâmicas grupais e lúdicas, com avaliação } \\
\text { por meio de pré e pós-teste, abordando temas sobre nutrição, higiene, prevenção de acidentes } \\
\text { domésticos, manejo da criança doente em casa e o desenvolvimento nos três primeiros anos de } \\
\text { vida das crianças, antes, imediatamente depois da intervenção e cinco meses após a intervenção. }\end{array}$ \\
\hline
\end{tabular}

Fonte: Autores. 


\section{Resultados e Discussão}

Após analisar os dados, foram identificados 10 (77\%) estudos nacionais e três (23\%) internacionais, conforme Quadro 3. Foi realizada a descrição das regiões do país, sendo o nordeste a região com maior número de publicações, ademais, um estudo não indicou região (NI).

Quadro 3: Distribuição dos estudos segundo País e Região.

\begin{tabular}{|c|c|c|c|}
\hline \multicolumn{2}{|c|}{ Nacionais } & \multicolumn{2}{|c|}{ Internacionais } \\
Nordeste & 6 & México & 1 \\
Norte & 1 & Portugal & 1 \\
Sul & 1 & Irã & 1 \\
Sudeste & 1 & & \\
NI & 1 & & \\
\hline
\end{tabular}

Fonte: Autores.

Se tratando ao tipo de estudo, dos 13 estudos encontrados, 10 (77\%) são originais, dois (15\%) são de revisão e um $(8 \%)$ relato de experiência. (Gráfico 1$)$

Gráfico 1: Descrição dos tipos de estudo.

\section{Tipo de estudo}

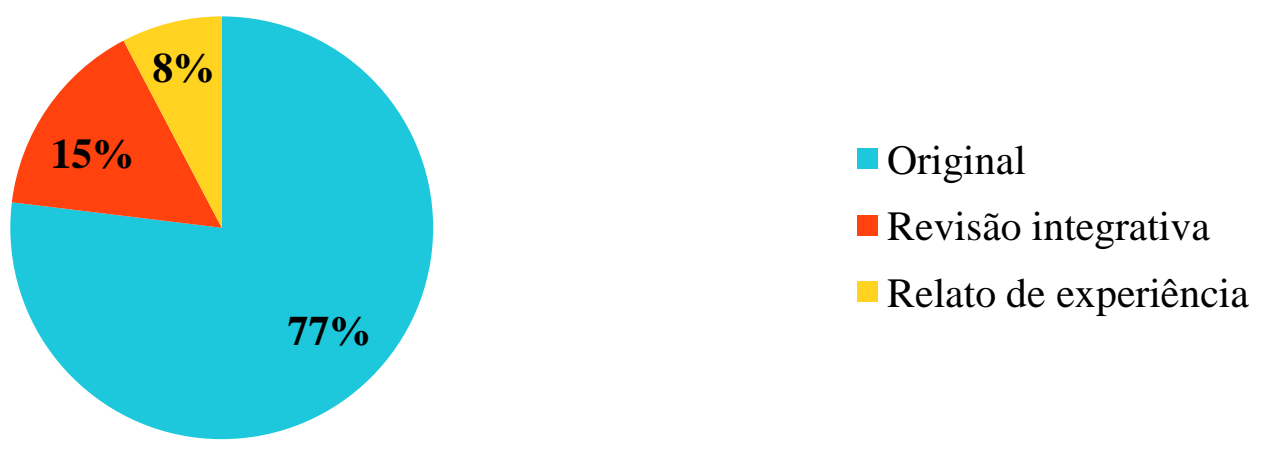

Fonte: Autores.

Foi identificado que o maior número de publicações foi no ano de 2017 (31 \%) seguidos de 2018 e 2019 (23\%), 2015 (15\%) e 2016 (8\%). Estudos de 2020 não foram incluídos no estudo. (Gráfico 2) 
Gráfico 2: Distribuição anual das publicações.

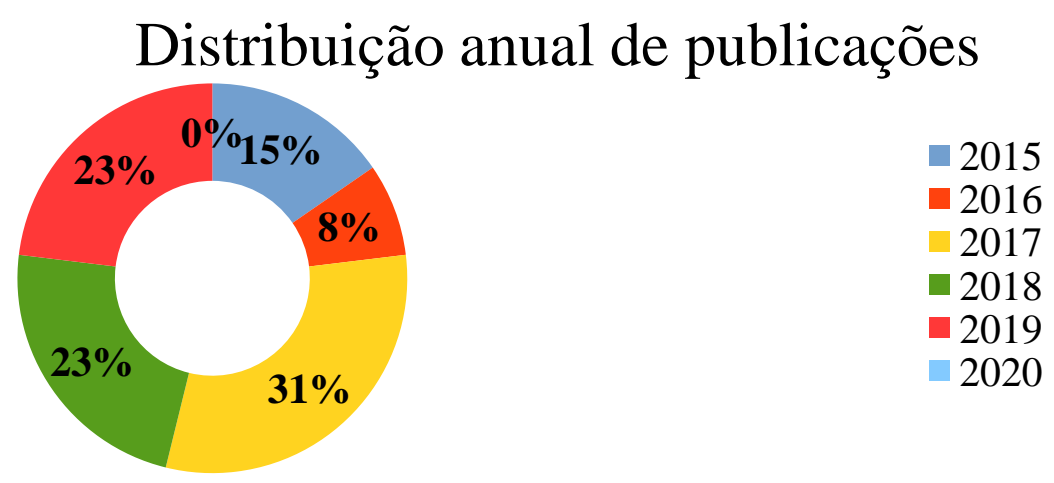

Fonte: Autores.

Inicialmente, foram identificados os principais ADI evidenciados nos estudos encontrados. Depois de lidos, consolidou-se que os principais ADI ocorridos no domicílio foram: quedas (67\%), seguido de queimadura (17\%), asfixia, corte e intoxicação (4\%), mordida de cachorro e afogamento (1\%). (Gráfico 3)

Gráfico 3: Principais acidentes domésticos na infância encontrados.

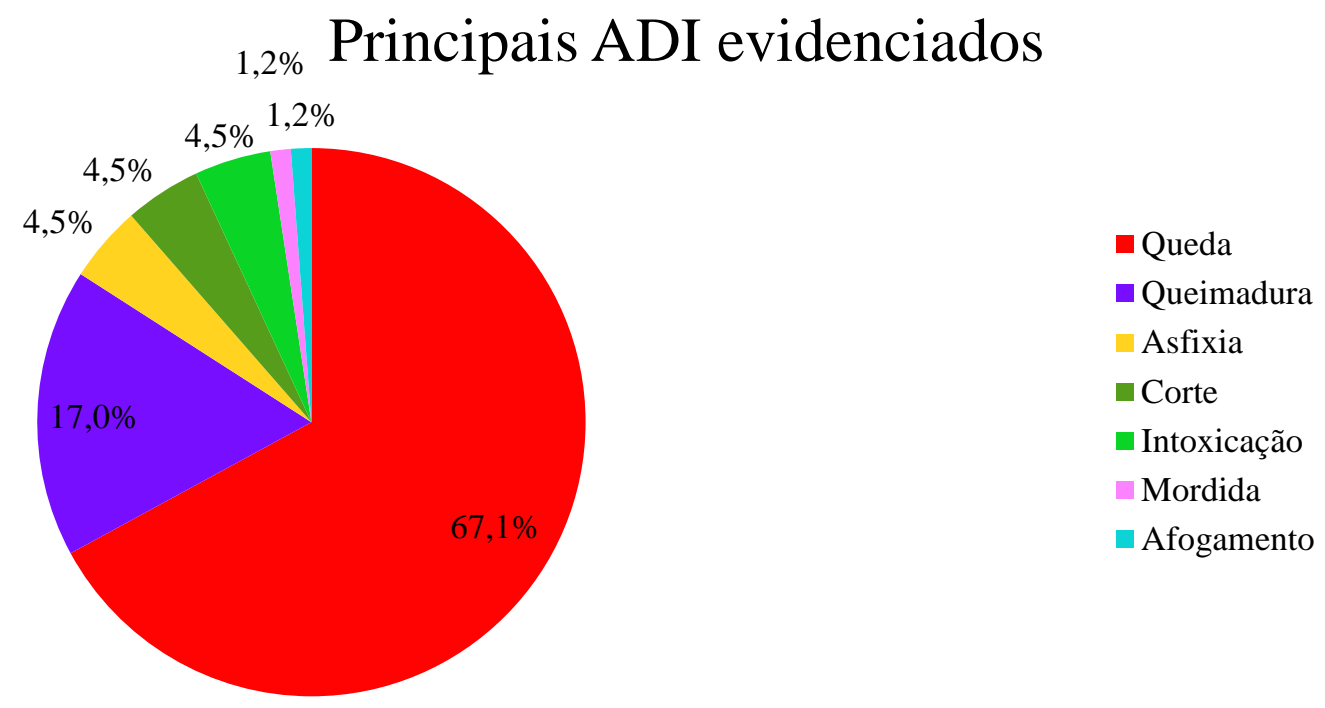

Fonte: Autores.

Uma vez encontrados os principais ADI, os próximos resultados foram divididos por categorias, sendo essas: Epidemiologia dos ADI, fatores de risco, perfil familiar e das crianças e a assistência de enfermagem na prevenção dos ADI.

\section{Acidentes Domésticos na Infância - Epidemiologia}

Os acidentes domésticos na infância são um importante problema para a saúde pública a nível mundial. Os achados desse estudo corroboram com outros, onde identificaram que a queda foi o principal acidente que compreende a faixa etária de zero a quatro anos (Brito et al., 2017) e que a queda foi a maior causa de procura em uma emergência relacionada a acidentes 
Research, Society and Development, v. 10, n. 9, e48110918027, 2021

(CC BY 4.0) | ISSN 2525-3409 | DOI: http://dx.doi.org/10.33448/rsd-v10i9.18027

domésticos (Brito \& Rocha 2015). Segundo dados da ONG Criança Segura, no ano de 2018, foram notificados 32.441 casos de hospitalização por queda (Correia et al., 2019). Os mecanismos predominantes dos traumas possuem uma essencial atenção, compreendendo que o essas situações podem resultar em diversas lesões, que envolvem de pequenos traumatismos até a morte. (Esfanjani et al., 2017). Cabe destacar que em relação às quedas, o quarto foi o local de maior acontecimento (48\%), seguido de sala (21\%), cozinha (17\%) e quintal (14\%). (Brito et al., 2017; Brito \& Rocha 2015; Esfanjani et al., 2017; Gomes 2015; Lima et al., 2019; Ribeiro et al., 2019 B; Silva et al., 2018). Segundo os estudos a face foi o segmento corporal mais atingida (47\%) seguido de membros inferiores (33\%) e membros superiores (20\%). (Brito et al., 2017; Esfanjani et al., 2017; Lima et al., 2019; Silva et al., 2018).

A queimadura se trata do segundo tipo de acidente mais comum encontrado nas literaturas selecionadas. Os estudos evidenciaram que a maior parte das internações infantis em centros de queimados, foram do sexo masculino (Correia et al., 2019; Esfanjani et al., 2017; Silva et al., 2017) e que 90\% ocorreram dentro do domicílio. (Correia et al., 2019; Silva et al., 2017). Um estudo iraniano identificou que as queimaduras foram o principal motivo de internações em crianças menores de sete anos. (Esfanjani et al., 2017). Líquidos quentes se mostraram como principal causa de queimadura em crianças e os custos hospitalares para crianças que sofreram esse tipo de acidente são os mais altos, comparados com outras lesões. (Correia et al., 2019; Esfanjani et al., 2017; Silva et al., 2017). As queimaduras de segundo e terceiro grau registram maior tempo internação. Em virtude das queimaduras, os membros superiores foram os locais mais afetados (Silva et al., 2017, Silva et al., 2018).

Havendo discordâncias de outros estudos que evidenciaram que membros inferiores são os principais segmentos afetados (Correia et al., 2019) Foram identificados a ocorrência de queimaduras a partir de condução elétrica, embora apareçam em menor número. Na maior parte dos casos o choque é de baixa voltagem e acontecem na ocorrência de fiações desencapadas e colocação de mão e objetos em tomadas (Correia et al., 2019; Silva et al., 2017). Enfatiza-se que um estudo Paulista identificou que mais da metade das crianças atendidas em um centro de queimados, desenvolveram infecção. Justificase essa condição devido ao comprometimento da principal barreira de proteção, que é a pele e devido a essa condição, o indivíduo se encontra mais vulnerável a invasão de microrganismos e descontrole hídricos (Francisco et al., 2013).

Se tratando dos outros ADI identificados, a asfixia é mais comum em crianças de até 96 dias, ocorrendo maioritariamente em meninos e de cor branca (Sales et al 2013). O corte e lacerações são descritas como consequência de outras condições, em especial as quedas (Brito et al., 2017) estudos evidenciaram que foi o tipo de lesão mais frequente. (Ribeiro et al., 2019; Silva et al., 2018). Segundo dados do SINITOX, o principal causador de intoxicação no ambiente doméstico são os medicamentos, contudo, a principal causa de óbitos na faixa etária entre 0 e 5 anos é a ingestão de produtos químicos. Se tratando das mordeduras, a faixa etária entre 5 e 9 anos é a mais comumente afetada, seguido de 0 a 4 anos. (Fiocruz 2017). Os cães foram os principais animais descritos e os membros inferiores são geralmente os mais afetados (Cardoso et al., 2018). O afogamento representa a segunda maior causa de acidente em menores de um ano e configura a principal causa de morte não intencional, crianças menores de 4 anos são mais propensas ao risco (Brito et al., 2017).

\section{Acidentes Domésticos na Infância - Fatores de Risco}

Se tratando do ambiente doméstico, os fatores de risco existentes podem prejudicar o desenvolvimento da criança, colaborando para a ocorrência de variados tipos de acidentes que, a depender da situação, podem resultar na ocorrência de casos graves e até danos irreparáveis (Brito et al., 2015).

Diversas são as causas que propiciam a ocorrência de ADI no ambiente doméstico. A rede alta, a presença de escadas ou degraus sem corrimão e saída e passagem condicionado com brinquedos, móveis, caixas ou outro objeto potencialmente obstrutivos, foram importantes variáveis identificadas na promoção a queda no ambiente doméstico (Brito et al., 2017). As 
crianças se desenvolvem a partir da exploração ativa do local a qual se encontra, em contrapartida, podem se ficar mais expostas ao risco de queda de modo mais frequente, pois se trata de uma idade de muita fragilidade devido à incapacidade de reconhecer riscos na realização de atividades (Ribeiro et al., 2019B).

As queimaduras são consideradas acidentes com grande risco de gravidade devido a questões sociais e individuais. São ditas como as lesões mais assoladoras que o corpo humano pode comportar (Correia et al., 2019). A cozinha é o ambiente doméstico com maior prevalência de queimaduras em crianças. A escaldadura e queimadura térmica são descritas como as mais frequentes, possuindo associação com o preparo das refeições (Paschoal et al., 2009). Por vezes, a má conservação dos equipamentos domésticos favorece possíveis acidentes no ambiente doméstico, além disso, a circulação de crianças na cozinha e a aproximação do fogão podem justificar a vasta frequência de queimaduras com líquidos quentes (Correia et al., 2019).

Quanto ao risco de asfixia, recém-nascidos são os mais susceptíveis a ocorrência (Sales et al., 2017) que se dá sobretudo, durante o sono onde, lençóis, pelúcias, grades com roupas de cama folgadas, são potenciais fatores de risco. Brinquedos, botões, acessórios de roupas e outros objetos pequenos também favorecem a ocorrência de asfixia. Até $75 \%$ dos casos de ingestão de corpos estranhos ocorrem em crianças com menos de cinco anos e que em $40 \%$ das ocorrências, não há acompanhante próximos (Simas \& Souza 2019).

Poucos estudos abordam sobre corte, intoxicação, mordedura e afogamentos. No que concerne corte e lacerações, a facilidade de acesso a materiais cortantes é um dos fatores de risco direto para a ocorrência (Ribeiro et al., 2019). Os produtos potencialmente causadores de intoxicação podem apresentar embalagens bem chamativas, atiçando o interesse da criança. Guardar esses produtos em locais inadequados, com facilidade no manuseio e acondicionamento em garrafas de refrigerantes, aparecem como os principais fatores de risco relacionados (Brito et al., 2015; Sanches et al., 2016). Quanto a mordeduras, a falta de supervisão e o não conhecimento do perfil e raça do animal se encontram como principais fatores de risco (Brito et al., 2015) e no que concerne afogamentos, $2 \mathrm{~cm}$ de água são suficientes para ocorrência de casos. A idade menor que 2 anos, ausência de barreira física em piscinas, baldes cheios sem supervisão de um adulto são os principais fatores de risco (Ribeiro $e t$ al., 2019).

\section{Acidentes Domésticos na Infância - Perfil Familiar}

Os achados desse estudo corroboram que os ADI são mais frequentes em crianças do sexo masculino (Batalha et al., 2016; Ribeiro et al., 2019; Silva et. al., 2017; Moita et al. 2018; Correia \& Silva 2006; Lima et al., 2013; Acker \& Cartana 2009; Karino \& Felli 2012) São diversas teorias e hipóteses existentes para justificar tal condição. Os meninos possuem maior propensão a se expor a brincadeiras arriscadas pois, por questões comportamentais e culturais, possuem maior liberdade quando comparados a meninas (Correia et al., 2017). Além disso, as brincadeiras são mais dinâmicas e requerem maior contato físico (Brito \& Rocha 2015). É apontado também, que meninos possuem menor vigilância e cuidados em jogos e atividades. (Silva et al., 2018).

Se tratando da faixa etária, o maior número de ADI ocorreu em menores de 5 anos. (Ribeiro et al., 2019; Silva et. al., 2017; Moita et al. 2018; Correia \& Silva 2006; Lima et al., 2013; Acker \& Cartana 2009; Karino \& Felli 2012). Acredita-se que as atividades recreativas das crianças dessa idade possuem relação com as particularidades do amadurecimento propiciado nessa faixa etária, que compreende a curiosidade, imaturidade e desprovimento de coordenação motora, facilitando situações de perigo (Brito et al., 2017). Há diferenças na ocorrência de ADI entre as fases Toodler e pré escolar. A fase toodler compreende a faixa etária entre 1 e 2 anos e 11 meses e é descrito como obtenção de habilidades motoras grossas e finas, tendo como principal competência, a locomoção, proporcionando maior domínio de atividades. A liberdade de locomoção aumentada somada a dificuldade de identificar perigos, são considerados fatores de riscos significativos. Já na fase pré-escolar, que 
compreende a faixa etária entre 3 e 5 anos, é identificado maior habilidade de coordenação, equilíbrio, atenção e identificação de situações perigosas (Brito \& Rocha 2015).

A partir dos estudos, identificou-se que há relação entre episódios de acidentes domésticos e a baixa renda familiar. A ocorrência de atendimentos chega a ser cinco vezes maior em crianças com menor nível socioeconômico. (Correia et al., 2017) A ausência de um espaço com tamanho adequado para lazer e brincadeiras da criança, (Lima et al., 2019) e a omissão dos relatos não graves (Silva et al., 2018). Além disso, tal condição dobra as chances de óbitos por acidentes dentro do domicílio quando comparados com as que possuem melhores condições financeiras (Brito \& Rocha 2015).

O nível de escolaridade foi um fator importante. Quanto maior o nível de instrução, maiores chances de identificação de riscos intradomiciliares, (Silva et al., 2018) em contrapartida, quanto menor conhecimento, menos importância à vigilância (Correia et al., 2017).

\section{Acidentes Domésticos na Infância - Assistência de Enfermagem}

O enfermeiro como profissional capacitado e elementar mediador na atenção básica, possui grande aproximação da população. Assim, fica sob sua incumbência a orientação e educação aos pais sobre os acidentes domésticos na infância e formas de prevenção (Passos \& Santos, 2016).

Fortalecer os conhecimentos junto aos pais e familiares, sobre a magnitude da prevenção dos ADI é de fundamental importância. Sobretudo, expor apenas formas de prevenção determinados agentes causadores de acidentes não é o suficiente. Para que tal condição seja efetiva, é necessário compreender a família como um todo, condições socioeconômicas, demográficas e culturais, condições de moradia e familiares. Tais elementos poderão impactar direta ou indiretamente na prevenção dos ADI (Lima et al., 2019).

Diante do conhecimento desses fatores, é possível desenvolver estratégias direcionadas, evidenciando os fatores de riscos, as medidas de segurança e finalmente, reduzir a incidência de ADI. Na atenção primária, desenvolver estratégias educacionais para alcance de pais e cuidadores, pode resultar na conscientização dos mesmos e mudanças no ambiente a fim de torná-lo mais seguro (Brito et al., 2017). Esses profissionais devem estar em constante atualização dos conhecimentos, uma vez que dúvidas possam surgir (Margotti et al., 2018).

Ressalta-se que a presença de adultos na hora dos acidentes nem sempre é fator protetivo para a ocorrência dos mesmos. Por isso, é necessário que além das orientações de prevenção, a educação sobre como agir diante de determinadas situações façam parte do planejamento. O eficiente conhecimento de pais e cuidadores sobre primeiros socorros e a correta maneira de agir, podem resultar na redução de danos (Sanches et al., 2016).

Em geral, quando falamos sobre educação da família, as técnicas educativas utilizadas se dão por meio de impressos, audiovisuais, demonstrações verbais e etc. Se tratando de educação em saúde, as pessoas recebem diversas informações a respeito e, normalmente, se deparam com dificuldades e barreiras na compreensão e o enfermeiro possui fundamental papel como facilitador da compreensão e retenção das instruções recebidas. Para isso, é necessário que o enfermeiro possua habilidade para identificar a assimilação de novas informações e a disposição para o aprendizado e o modo que facilite a compreensão, enfatizando-se a necessidade de novos estudos para análise e quantificação da compreensão das pessoas (Francisco et al., 2013).

\section{Conclusão}

A análise dos estudos evidenciou um grande número de ocorrência de ADI e tais condições refletem em importantes repercussões emocionais e físicas, seja na criança quanto na família. 
Verificou-se que as quedas e queimaduras constituem as principais causas de acidentes, a faixa etária predominante foi de menores de 5 anos e os meninos são mais susceptíveis. Segundo os estudos, o quarto foi o local mais comum em relação às quedas e quanto a queimaduras, líquidos quentes se mostraram como principal agente causador. Além disso, queimaduras elétricas vêm sendo cada vez mais frequentes.

Diversos são os fatores de risco presentes no ambiente doméstico, todavia, muitos desses fatores podem ser reduzidos e até eliminados, a partir da conscientização dos pais e cuidadores. A baixa renda familiar e o baixo nível de escolaridade possuem importante papel na ocorrência de ADI.

O enfermeiro possui essencial atuação na educação de pais e familiares na prevenção dos ADI, além disso, educação de primeiros socorros podem evitar complicações. Conhecer questões sociais e de moradia são importantes alternativas no desenvolvimento de estratégias para redução de riscos, pois diante de tais informações, o profissional pode adequar a abordagem ao familiar.

Diante do exposto, é necessário que o tema seja expandido e novos estudos sejam realizados, em especial sobre a identificação dos fatores sociais e fragilidades no conhecimento de pais e familiares a respeito da prevenção dos ADI.

\section{Referências}

Acker, J. I. B. V. \& Cartana, M. H. F. (2009) Construção da participação comunitária para a prevenção de acidentes domésticos infantis. Rev. bras. enferm. 62(1): $64-70$.

Batalha, S., Salva, I., Santos J., Albuquerque C., Cunha F., Sousa H. (2016) Acidentes em Crianças e Jovens, Que Contexto e Que Abordagem? Experiência de Nove Meses no Serviço de Urgência num Hospital de Nível II. Acta Pediátrica Portuguesa, 7, -37.

Brasil. Ministério da Saúde. (2012) Óbitos entre crianças de até 10 anos caem 31\%. http://www.blog.saude.gov.br/promocao-da-saude/31601-obitos-entrecriancas-de-ate-10-anos-caem-31

Brito, M. A., Melo, A. M. N., Veras, I. C., Oliveira, C. M. S., Bezerra, M. A. R., Rocha, S. S. (2017) Fatores de risco no ambiente doméstico para quedas em crianças menores de cinco anos. Rev. Gaúcha Enferm. 38( 3 )

Brito, M. A. \& Rocha, S. S. (2015) A Criança Vítima de Acidentes Domésticos Sob o Olhar das Teorias de Enfermagem. J. res.: fundam. care. Online 7(4): 3351-65.

Cardoso, E., Andriani, C., Hachmann, L., Catafesta, B., Venzon, J. C. (2018) Características Epidemiológicas De Acidentes Por Mordeduras De Animais No Município De Indaial - SC. Rev. Civet. 5(2):148-64.

Correa, I. \& Silva, F. M.(2006) Prevenção de acidentes domésticos à criança menor de 5 anos: percepção materna. REME - Rev. Min. Enf.,10(4):397-401, out./dez., 2006

Correia, D. S., Chagas, R. R. S., Costa, J. G., Oliveira, J. R., França, N. P. A., Taveira, M. G. M. M. (2019) Perfil de crianças e adolescentes internados no centro de terapia de queimados. Revista de Enfermagem UFPE on line, 13(5): 1361-1369.

Esfanjani, R. M., Bazargani, H. S., Golestani, M., Mohammadi, R. (2017) Domestic Injuries among Children Under 7 Years of Age in Iran, The Baseline Results from the Iranian First Registry. Bull Emerg Trauma. 5(4): 280-284.

Francisco, T., Nóbrega, S., Valente, R., Santos, M., Pereira, G., Estrada, J., Serafi, Z., Ventura, L. (2013) Grande queimado numa Unidade de Cuidados Intensivos Pediátricos - experiência de 20 anos. NASCER E CRESCER: revista de pediatria do centro hospitalar do porto, XXII

Fundação Oswaldo Cruz. Instituto de Comunicação e Informação Científica e Tecnológica em Saúde. (2017) Sistema Nacional de Informações Tóxico-Farmacológicas (SINITOX). Casos e óbitos registrados de intoxicação humana e envenenamento: faixa etária. Rio de Janeiro: Fiocruz.

Gomes, L. M. X., Rocha, R. M., Barbosa, T. L. A. \& Silva, C. S. O. (2013) Descrição dos acidentes domésticos ocorridos na infância. O Mundo da Saúde, 4, 394-400.

Gómez, O. S. M. (2015) Prevalencia de accidentes en el hogar en niños y factores de riesgo asociados. Enferm. Univ. 12( 3 ): $116-121$.

Karino, M. E. \& Felli, V. E. A. (2012) Enfermagem baseada em evidências: avanços e inovações em revisões sistemáticas. Cienc. Cuid. Saúde.11(5):011-015.

Lima, E. P. M., Almeida, A. O. A., Beserra, E. P., Carneiro, E. P., Andrade, F. M. R. \& Gubert, F. A. (2018) Identificação dos conhecimentos de mãos na prevenção de acidentes domésticos com crianças da primeira infância. Enferm. Foco 2018, 9 (4): 77-80.

Lima, D. B., Moura, J. C., Tirico, S. H. N., Mazzeo, M. R., Cunha, M. T., Sperandio, R. A., Silberfeld, M., Simone, M. C., Bibikoff, S., Tambellini, E. F. \& Marsiglia, R. M. G. (2013) Promoção à saúde e prevenção de acidentes na infância: uma ação de estudantes de medicina. Rev Med, 92(2):119-27. 
Research, Society and Development, v. 10, n. 9, e48110918027, 2021 (CC BY 4.0) | ISSN 2525-3409 | DOI: http://dx.doi.org/10.33448/rsd-v10i9.18027

Margotti, E., Costa, P. P. S. \& Corrêa, A. M. C. (2018) A importância da prevenção de acidentes na infância: Um relato de experiência. Rev Enferm Atenção Saúde [Online], 7(1): 200-208.

Moita, C. E., Andrade, A. M. S. \& Campos, R. C. G. (2018) Educação em saúde para prevenção de acidentes domésticos na infância. Revista de Trabalhos Acadêmicos. 1(5).

Paschoal, S.R.G., Pereira, D.M. \& Nascimento, E.M. (2009) Efect of an educative action on relatives knowledge about childhood burns at home. Rev Latinoamericano Enfermagem, 17(3), 341-346

Passos, D. A. \& Santos, W. L. (2016) O enfermeiro como educador para a prevenção dos principais acidentes ocorridos na primeira infância. Rev. Cient. Sena Aires, 5(2): 124-35.

Ribeiro, A., Barros, M., Pereira, I. A., Lírio, C., Pais, I. P. \& Couto, M. L. (2019) Conhecimentos e práticas parentais sobre medidas preventivas de acidentes domésticos e de viação. Revista Portuguesa de Medicina Geral e Familiar [série na Internet]. 1, 35(3). 186-95

Ribeiro, M. G. C., Paula, A. B. R., Bezerra, M. A. R., Rocha, S.S., Avelino, F. V. S. D. \& Gouveia, M. T. O. (2019) Determinantes sociais da saúde associados a acidentes domésticos na infância: uma revisão integrativa. Rev. Bras. Enferm. [Internet], 72(1): 265-276.

Sales, C. C. F., Suguyama, P., Guedes, M. R. J., Borghesan, N. B. A., Higarashi, I. H. \& Oliveira, M. L. F. (2017) Intoxicação na primeira infância: socorros domiciliares realizados por adultos. Rev baiana enferm. 31(4): e23766

Sanches, P. H. S., Sanches, J. A., Nogueira, M. J., Perondi, N. M., Sugai, M. H., Justulin, A. F., Vantine, G. R., Neto, O. T. (2016) Perfil epidemiológico de crianças atendidas em uma Unidade de Tratamento de Queimados no interior de São Paulo. Rev Bras Queimaduras,15(4):246-250.

Silva, M. F., Fontinele, D. R. S., Oliveira, A. V. S., Bezerra, M. A. R. \& Rocha, S. S. (2017) Determining factors of domestic accidents in early childhood. J Hum Growth Dev. 27(1): 10-18.

Silva, F. B., Gondim, E. C., Henrique, N. C. P., Fonseca, L. M. M. \& Mello, D. F. (2018) Intervenção educativa com mães jovens: aquisição de saberes sobre cuidados da criança. Acta paul. enferm. [Internet] 31(1): 32-38.

Simas, V. F. C \& Souza, A. S. (2019) Crianças hospitalizadas vítimas de acidentes na primeira infância. Revista Pró-UniverSUS. 10 (1): $25-28$.

Teis, M. A. \& Teis, D. T. (2006) A Abordagem Qualitativa: A Leitura no Campo de Pesquisa. 8p. http://bocc.ubi.pt/_esp/autor.php?codautor=969

Ursi, E. S. \& Gavão, C. M. (2006) Prevenção de lesões de pele no perioperatório: revisão integrativa da literatura. Rev. Latino-Am. Enfermagem [Internet]. 14(1): 124-131. 